\title{
Tuberkuloserisiko einfach im Blutbild ablesen
}

\author{
Wie können Personen identifiziert werden, die ein besonders hohes Risiko für die Entwicklung \\ einer aktiven Tuberkulose haben? Eine Studie aus Madagaskar weist uns den Weg.
}

_Der Tuberkulin-Hauttest wird einge-
setzt, um Personen mit latenter Tuber-
kulose - schätzungsweise ein Drittel der
Weltbevölkerung - zu identifizieren. Sie
profitieren von einer präventiven tuber-
kulostatischen Therapie, da etwa $10 \%$
von ihnen über Jahrzehnte auch eine ak-
tive Tuberkulose entwickeln.
In einer prospektiven longitudinalen
Kohortenstudie in Antananarivo auf
Madagaskar wurden 296 asymptomati-
sche Personen mit negativem Rönt-
genthorax untersucht. Sie lebten im glei-
chen Haushalt wie Personen mit nach-
gewiesener aktiver Tuberkulose. 186
Personen ohne derartigen engen Kon-
takt dienten als Kontrollen
18 Monate lang wurden die Teilneh-
mer regelmäßig untersucht. Bei Auftre-
ten von Tuberkulose-Symptomen oder
einem hochpositiven Tuberkulin-Haut-
test wurde in einem Tuberkulosezent-
rum nochmals ein Röntgenthorax ge- macht. Teilnehmer mit gesicherter Diagnose wurden tuberkulostatisch behandelt. Am Ende der Follow-up-Periode hatten 12 von 289 auswertbaren Kontaktpersonen $(4,4 \%)$ Symptome einer aktiven Tuberkulose entwickelt. Neun davon waren Kinder.

Der BCG-Impfstatus hatte keine Auswirkung auf den Tuberkulintest. Ein positiver Test korrelierte nicht mit dem Fortschreiten zur aktiven Tuberkulose. Allerdings waren Tuberkulose-Symptome signifikant mit einem Anstieg der Monozyten im Differenzialblutbild assoziiert (adjustierte Hazard Ratio: 6,25). Bei hochpositivem Tuberkulin-Hauttest mit einem Durchmesser des Infiltrats $\geq 14 \mathrm{~mm}$ und zusätzlich erhöhten Monozyten lag die Hazard Ratio sogar bei 8,46 (95\%-Konfidenzintervall: 1,74-41,22).

- Rakotosamimanana N et al. Biomarkers for risk of developing active tuberculosis in contacts of TB patients: a prospective cohort study. Eur Respir J. 2015;46:1095-103

\section{KOMMENTAR}

Für Asylsuchende ist das Screening auf Tuberkulose vorgeschrieben. Die Thorax-Röntgenuntersuchung bleibt für das RobertKoch-Institut trotz eines kürzlichen Reviews die Methode der Wahl für Personen ab 16 Jahren. Es erscheint fraglich, ob wir diesen hohen Standard angesichts des Flüchtlingszustroms aufrechterhalten können. Vielleicht müssen wir einfachere Untersuchungen vorschalten, wie sie uns die hier vorgestellte Studie aus einem Drittweltland aufgezeigt hat. Unter Beachtung von Tuberkulintest und Differenzialblutbild ist eine relativ gute prädiktive Aussage der Progression von einer latenten Infektion zu einer aktiven Tuberkulose möglich. Wahrscheinlich müssen wir angesichts der schieren Zahl der Flüchtlinge aus Hochrisikogebieten Abschied nehmen vom gesundheitspolitischen Perfektionismus und uns auf einfachere Verfahren besinnen. Madagaskar macht es vor.

Prof. Dr. med. H. S. Füeß।

\section{Eine Sechsjähriger mit Wimpernläusen}

Ein sechsjähriger Junge wurde in der Universitätsklinik von Catania wegen anhaltender Rötung und Juckreiz im Bereich der Augenlider (Abb. A) vorgestellt. Unter der Diagnose einer atopischen Dermatitis war er bereits zwei Wochen lang mit kortikoidhaltigen Salben und Antihistaminika erfolglos behandelt worden. Bei der Dermatoskopie erkannte man mehrere Filzläuse (Phthirus pubis) sowie ovaläre Nissen an den Wimpern (Abb. B). Unter der Diagnose einer Phthiriasis palpebrarum wurde der Junge mit 5\%iger gelber Quecksilberoxidsalbe viermal am Tag über zwei Wochen hinweg behandelt, worunter sich die Symptomatik vollständig zurückbildete.

Die Wimpern sind bei Kindern ein übliches Habitat für die Filzlaus, nachdem sich in diesem Alter an anderen Körperstellen noch kein Terminalhaar findet. Die Läuse werden üblicherweise durch engen Kontakt mit befallenen Erwachse-
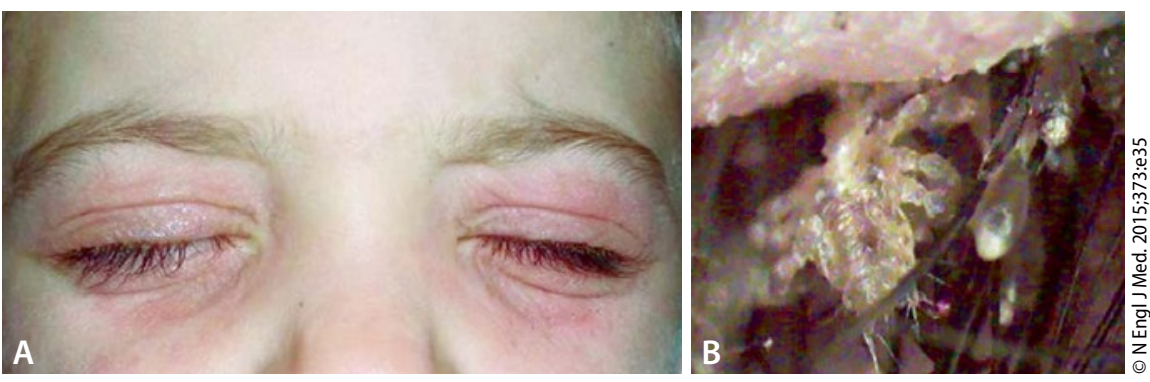

A: Beidseitige Rötung im Bereich der Augenlider, B: Filzläuse (Phthirus pubis) und ovaläre Nissen an den Wimpern.

nen übertragen. Bedenkt man die Lokalisation bei Erwachsenen, so keimen heutzutage natürlich dunkle Gedanken über den Übertragungsmechanismus auf. Die Diagnose ist manchmal optisch nicht leicht zu stellen, da sich die Läuse tief in den Lidrand eingraben und man wegen der Rötung im Bereich der Augen zunächst an eine atopische Dermatitis oder eine allergische Konjunktivitis denkt.

Prof. Dr. med. H. S. FüeßI

- Micali G, Lacarubba F (cldermct@gmail.com). Phthiriasis palpebrarum in a child. N Engl J Med. 2015;373:e35 